\title{
The non-clonality of drug resistance in Beijing- genotype isolates of Mycobacterium tuberculosis from the Western Cape of South Africa
}

\author{
Thomas R loerger ${ }^{1 *}$, Yicheng Feng ${ }^{2}$, Xiaohua Chen ${ }^{2}$, Karen M Dobos ${ }^{3}$, Thomas C Victor ${ }^{4}$, Elizabeth M Streicher ${ }^{4}$, \\ Robin M Warren ${ }^{4}$, Nicolaas C Gey van Pittius ${ }^{4}$, Paul D Van Helden ${ }^{4}$, James C Sacchettini ${ }^{2}$
}

\begin{abstract}
Background: The Beijing genotype of M. tuberculosis is a virulent strain that is disseminating worldwide and has a strong association with drug resistance. In the Western Cape of South Africa, epidemiological studies have identified the R220 cluster of the Beijing genotype as a major contributor to a recent outbreak of drug-resistant tuberculosis. Although the outbreak is considered to be due to clonal transmission, the relationship among drug resistant isolates has not yet been established.

Results: To better understand the evolution of drug resistance among these strains, 14 drug-resistant clinical isolates of the Beijing genotype were sequenced by whole-genome sequencing, including eight from R220 and six from a more ancestral Beijing cluster, R86, for comparison. While each cluster shares a distinct resistance mutation for isoniazid, mapping of other drug-resistance mutations onto a phylogenetic tree constructed from single nucleotide polymorphisms shows that resistance mutations to many drugs have arisen multiple times independently within each cluster of isolates. Thus, drug resistance among these isolates appears to be acquired, not clonally derived. This observation suggests that, although the Beijing genotype as a whole might have selective advantages enabling its rapid dissemination, the XDR isolates are relatively less fit and do not propagate well. Although it has been hypothesized that the increased frequency of drug resistance in some Beijing lineages might be caused by a mutator phenotype, no significant shift in synonymous substitution patterns is observed in the genomes.
\end{abstract}

Conclusion: While MDR-TB is spreading by transmission in the Western Cape, our data suggests that further drug resistance (i.e. XDR-TB) at this stage is acquired.

\section{Background}

The Beijing genotype of Mycobacterium tuberculosis is a virulent strain that originated out of East Asia [1] and has disseminated around the world [2,3]. It is a member of principle genetic group 1 [4], bearing the markers KatG 463Leu and GyrA 95Thr, and is characterized by a spoligotype 000000000003771. Isolates of the Beijing strain have been associated with decreased survival times in mice [5-7] and in the rabbit meningitis model [8], and increased growth rates in human macrophages $[9,10]$. Some patients infected with Beijing genotype

\footnotetext{
* Correspondence: ioerger@cs.tamu.edu

'Department of Computer Science and Engineering, Texas A\&M University, College Station, TX USA

Full list of author information is available at the end of the article
}

show increased radiographic cavitation [11,12] and experience more treatment failures, independent of differences in drug resistance [13]. One potential explanation for the increased virulence is the production of phenolglycolipid (PGL), a surface antigen that suppresses the Th1 response [14]. PGL is produced in Beijing strains, but is not produced by members of the other principle genetic groups (2 and 3), such as M. tuberculosis $\mathrm{H} 37 \mathrm{Rv}$, because the polyketide synthase pks15/1 has a frameshift mutation splitting it into two separate open reading frames (ORFs) [15].

The Beijing genotype is strongly associated with drug resistance [16], including multi-drug resistance (MDR$\mathrm{TB})$ and extensive-drug resistance (XDR-TB). One of the first outbreaks of MDR-TB, which occurred in

\section{Biomed Central}


New York City in the early 1990's, was found to be a clonal expansion of a variant of the W-Beijing strain $[17,18]$. The Beijing strain was also associated with an outbreak in Azerbaijan prisons, where nearly all TB infections were Beijing, and $>50 \%$ were MDR [19]. The Beijing genotype has been reported to account for $34 \%$ of the XDR cases across South Africa [20]. Similar findings of increased association of drug resistance with strains of the Beijing genotype have been reported in India [21], Russia [22], Korea [23], Vietnam [24], Japan [25], and Germany [26]. In a large-scale study that included both civilians and prison inmates in Russia, $67 \%$ of the TB infections were Beijing, but the frequency of resistance to drugs like isoniazid, rifampicin, streptomycin, and ethambutol was nearly twice as high among Beijing as non-Beijing isolates [11]. Although these studies were performed in different populations using varying methodologies, taken together, they support the general view that infections with the Beijing strain are more likely to be drug resistant than other strains of TB.

Currently, the Beijing strain constitutes a significant component of a major outbreak of TB in the Western Cape of South Africa, where it represents $36.5 \%$ of the drug-resistant cases (in a sample between 2005 and 2006 [27]). The proportion of the Beijing genotype among drug-resistant cases is inflated relative to the overall proportion of Beijing strains among drugsusceptible TB cases in the region, which was estimated at 21.9\% in Cape Town (between 1993 and 2004) [28]. In another study of $\mathrm{TB}$ cases in the Western Cape region between 2001 and 2002, 28\% of drug-resistant cases were of the Beijing genotype, whereas $17 \%$ of cases overall were Beijing [29]. Epidemiological studies suggest that the Beijing genotype is highly transmissible (based on geographical clustering of individual strains within households and communities [30]), leading to a hypothesis that drug resistance is spreading clonally through the region. However, the clonal expansion hypothesis contrasts with most TB outbreaks, which are often found to be constituted of a mixture of genotypes [31]. In fact, drug resistant mutations often incur a relative fitness cost, making it more difficult for them to compete [32]. van der Spuy et al. [28] found that the increase in Beijing strains in Cape Town was primarily due to drug-susceptible strains. Over a 12-year period, the number of drug-susceptible Beijing isolates increased exponentially with a doubling time of $\sim 4$ years, whereas the frequency of most other genotypes, including drugresistant Beijing isolates, remained relatively constant. This observation was taken to suggest that the success of the Beijing strain is due overall to increased virulence rather than transmissibility [28], and that drug-resistant strains of Beijing were less fit that drug-susceptible Beijing strains. Thus the association of Beijing with drug- resistance could be a side-effect due to the increased overall success of this strain.

The Beijing genotype family can be divided into seven lineages, progressing from "ancestral" (sublineage 1) to "modern" (sublineage 7). These distinctions are based on comparison of IS6110 insertion sites, regions of deletion, and other markers [33]. Sublineage 7 is most prevalent in the Western Cape $(72.6 \%$ in Cape Town between 1993 and 2004), which, discounting founder effects, is interpreted to mean that it has higher fitness [33]. Some studies have suggested that different lineages have different propensities to develop drug resistance. For example, Mokrousov et al. [34] found that ancestral lineages in China had a higher frequency of resistance to rifampicin and pyrazinamide. However, these findings were contradicted by a larger study of Beijing isolates in South Africa that found no statistically significant difference in drug resistance among the seven lineages [33].

One particular sub-group of the Beijing family has recently been identified, cluster R220 (based on IS6110 RFLP banding patterns), that is overrepresented among drug-resistant isolates in the Western Cape of South Africa [27]. R220 is a member of sublineage 6, representing a modern Beijing variant. R220 constituted over $75 \%$ of isolates of the Beijing clade in 2005-2006 in the Western Cape, and accounted for $42 \%$ of the increase in drug-resistant cases since 2001 [27]. R220 was also found to be prevalent among children infected with drug-resistant TB in the region [35]. Cluster R220 strains share the same c-15t inhA promoter mutation responsible for resistance to isoniazid and many isolates (nearly 75\%) also have the Ser531Leu (TTG) rpoB conferring rifampicin resistance, suggesting that it has evolved into a distinct MDR clone. If it were a distinct clone, this might imply that it has acquired compensatory mutations that enable it to tolerate the fitness cost associated with drug resistance mutations or enhance its transmissibility in the population.

Although R220 is a well-defined cluster in terms of spoligotyping and IS6110 RFLP fingerprinting, these are still coarse-grained methods of genotyping and do not guarantee that the drug-resistant isolates in this cluster are clonally derived. In order to get a more fine-grained picture of the R220 cluster, we performed whole-genome sequencing on eight drug-resistant isolates of R220 from various locations in the Western Cape of South Africa. For comparison, we also sequenced HN878, a drug-susceptible isolate from the US, and six drugresistant isolates of the $\mathrm{R} 86$ cluster, which is part of the more ancestral sublineage 1 . Using these whole-genome sequences, we reconstruct a phylogenetic tree and map drug-resistance mutations on them to examine the hypothesis of clonality. 


\section{Methods}

\section{Selection of Strains for Sequencing}

M. tuberculosis HN878 was obtained from the NIH TB Vaccine Testing and Research Materials Laboratory at Colorado State University. Fourteen additional drugresistant strains of the Beijing genotype were selected for whole-genome sequencing and comparative analysis. The isolates were sampled from a database maintained at the DST/NRF Centre, Stellenbosch University, representing drug resistant TB cases from a variety of hospitals in the Western Cape, South Africa (see Table 1). The samples were all selected to be isoniazid-resistant, and were chosen to span a range of degrees of drug resistance, including cases that are mono-resistant (isoniazid), MDR (isoniazid and rifampicin), pre-XDR (MDR plus resistance to either a fluoroquinolone or an aminoglycoside), and XDR. No other biases (e.g. age, gender, HIV status) were applied in the sampling. All 14 isolates have the Beijing spoligotype, 00000000003771. Six of the isolates investigated in this study are from sublineage 1, representing the atypical Beijing genotype (ancestral, family F31). IS6110-RFLP analyses have classified these strains as being members of cluster R86. The remaining 8 isolates are from cluster R220 in sublineage 6 , which represents "typical" Beijing genotype strains (more recently evolved). The IS6110 RFLP patterns used for determining cluster membership of these isolates are shown in Figure 1.

\section{Drug-Susceptibility Testing}

Drug susceptibility testing for isoniazid $(0.2 \mu \mathrm{g} / \mathrm{ml})$, rifampin $(1 \mu \mathrm{g} / \mathrm{ml})$, ethambutol $(7.5 \mu \mathrm{g} / \mathrm{ml})$, ethionamide $(20 \mu \mathrm{g} / \mathrm{ml})$, amikacin $(30 \mu \mathrm{g} / \mathrm{ml})$ and ofloxacin $(2 \mu \mathrm{g} / \mathrm{ml})$ was performed using the indirect proportion method on

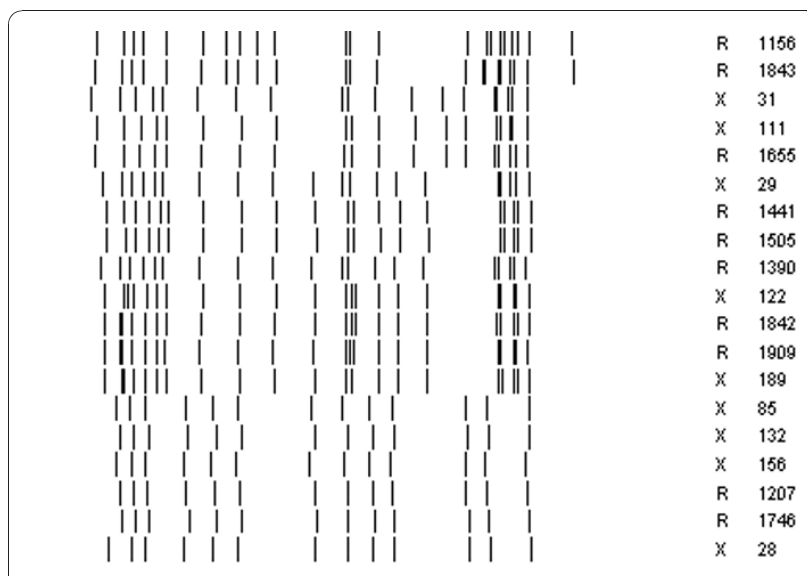

Figure 1 IS6110 RFLP fingerprints of the Beijing clinical isolates sequenced in this study.

Middlebrook's medium [36]. Additional drug susceptibility testing was done using the proportion method on Middelbrook's 7H10 medium containing ofloxacin, amikacin, kanamycin and capreomycin at the critical concentration of $2 \mu \mathrm{g} / \mathrm{ml}, 5 \mu \mathrm{g} / \mathrm{ml}, 5 \mu \mathrm{g} / \mathrm{ml}$ and $10 \mu \mathrm{g} / \mathrm{ml}$, respectively.

\section{DNA Preparation and Sequencing Reaction}

Sequencing of the genomes of 15 Beijing strains of $M$. tuberculosis was carried out on an Illumina Genome Analyzer II (Illumina, Inc.). In this study, Illumina Paired-End sequencing method (PE) was used. The cetyltrimethylammonium bromide (CTAB)-lysozyme method was used for extraction and purification of genomic DNA [37]. Suitable genomic DNA samples for the GAII were prepared as described on the sample preparation protocol (Illumina). 2-3 $\mu \mathrm{g}$ of genomic DNA

Table 1 Beijing strain clinical isolates from South Africa subjected to whole-genome sequencing

\begin{tabular}{|c|c|c|c|c|c|c|c|}
\hline Strain ID & Origin (hospital) & Sub-lineage & Family & Cluster & DR type & $\begin{array}{l}\text { Combined resistance } \\
\text { profiles determined by DST }\end{array}$ & $\begin{array}{l}\text { Combined sensitivity profiles } \\
\text { determined by DST }\end{array}$ \\
\hline R1207 & George & 1 & F31 & R86 & MDR & INH, RIF & EMB \\
\hline $\mathrm{X} 132$ & Khayelitsha & 1 & F31 & R86 & pre-XDR & INH,RIF,AMI,CAP,STR,KAN & ETH,OFL,EMB \\
\hline$X 28$ & Dysselsdorp & 1 & F31 & R86 & XDR & INH,RIF,AMI,CAP,OFL,STR, KAN & $\mathrm{ETH}, \mathrm{EMB}$ \\
\hline $\mathrm{R} 1746$ & Mossel Bay & 1 & F31 & R86 & MDR & $\mathrm{INH}, \mathrm{RIF}$ & EMB \\
\hline X156 & Brooklyn Chest & 1 & F31 & R86 & pre-XDR & INH,RIF, AMI,CAP,STR,KAN & ETH,OFL,EMB \\
\hline X85 & George & 1 & F31 & R86 & XDR & INH,RIF, AMI,OFL,KAN,ETH & CAP,EMB \\
\hline R1909 & Worcester & 6 & $\mathrm{~F} 29$ & $\mathrm{R} 220$ & MDR & INH,RIF,EMB & \\
\hline R1842 & George & 6 & F29 & $\mathrm{R} 220$ & Mono & $\mathrm{INH}$ & RIF, EMB \\
\hline $\mathrm{X} 122$ & Huguenot & 6 & F29 & $\mathrm{R} 220$ & pre-XDR & INH,RIF,OFL & ETH,AMI,EMB \\
\hline $\mathrm{R} 1390$ & Stellenbosch & 6 & F29 & $\mathrm{R} 220$ & Mono & $\mathrm{INH}$ & RIF,EMB \\
\hline X189 & Victoria Hos. & 6 & F29 & $\mathrm{R} 220$ & XDR & INH,RIF,AMI,CAP, OFL,KAN, & ETH,STR \\
\hline R1505 & George & 6 & F29 & $\mathrm{R} 220$ & MDR & INH, RIF & EMB \\
\hline R1441 & George & 6 & F29 & $\mathrm{R} 220$ & Mono & INH & RIF,EMB \\
\hline X29 & Retreat & 6 & $\mathrm{~F} 29$ & $\mathrm{R} 220$ & pre-XDR & INH,RIF,AMI,STR,KAN & EMB,OFL,CAP,ETH \\
\hline
\end{tabular}

$\mathrm{INH}=$ isoniazid $\mathrm{RIF}=$ rifampicin STR = streptomycin EMB = ethambutol ETH = ethionamide AMI = amikacin KAN = kanamycin CAP = capreomycin $\mathrm{OFL}=$ ofloxacin 
was used for sample preparation. Genomic DNA was sheared by a nebulizer to generate DNA fragments for the Illumina Paried-End Sequencing method. The specific oligonucleotides (Illumina adapters) designed for PE sequencing were ligated to both ends of DNA fragments with the TA cloning method. Adapter-ligated DNA fragments of length 350-400 bp were isolated from a $2 \%$ agarose gel (Certified low-range Ultra Agarose, BIORAD) by using QIAquick Gel Extraction Kit (QIAGEN). Then the fragments were amplified by PCR reaction to generate the DNA library (15-30 $\mathrm{ng} / \mu \mathrm{l})$. The median size of the library was estimated by examining the $2 \%$ agarose gel image. The molarity of the DNA library was estimated as described in the sample preparation protocol (Illumina). The DNA libraries (5 picomoles each), including the $\varphi$ X174 control (bacteriophage DNA), were loaded on the flow cell for the cluster generation and sequencing. 79 cycles of images were collected, representing two 36-bp reads (paired ends) plus 7 bases representing id tags for multiplexing. The images were analyzed using version 1.4 of the GAPipeline software supplied by Illumina, producing files with 10-20 million pairs of $36 \mathrm{bp}$ reads for each genome.

\section{Sequence Determination and Bioinformatics}

The reads were analyzed by comparative genome assembly to determine the complete sequence of each genome using custom software developed in our lab. The reads were first mapped (aligned) against the genome of $\mathrm{H} 37 \mathrm{Rv}$ as a reference sequence. The mapping of each read was accomplished by ungapped alignment to the reference genome (including the reverse complement strand), allowing at most two mismatches out of 36 nucleotides. Initially, the reads that were pair-mates were mapped independently; subsequently, locations of reads for which the paired-end did not match within $300 \mathrm{bp}$ were discarded. The mapped reads were used to assemble a list of the nucleotides observed at each position within the reference genome contributed by all the reads that overlapped it. Base calls were made by a maximum likelihood calculation, computed as the product of the probabilities for each base at each position using uncertainties estimated during image analysis. Sites where apparent differences were observed were subjected to local contig-building, in order to determine whether the difference was due to a nucleotide substitution or a small insertion or deletion.

For each genome, a list of verified differences was prepared and used to modify the reference genome to produce an intermediate ('edited') genome. Then the process was repeated by re-mapping the reads against the edited genome, and re-calling nucleotides at each position. For any sites that still had zero coverage, the base from the reference strain was used. This primarily included regions with exceptionally high GC-content (80-90\%).

Large-scale deletions were identified by analyzing paired-end data for reads whose paired-end maps an unusually long distance away (i.e. $>300 \mathrm{bp}$, compared to average read length of $\sim 200$ ). Large-scale insertions were determined by building contigs that spanned fragments, neither of whose paired ends mapped into the H37Rv genome. These $\sim 200 \mathrm{bp}$ fragments were then assembled into larger contigs using Newbler (software from Roche, inc.), localized in the genome using pairedend information, and identified by performing BLAST searches on the NCBI website.

Virtual spoligotyping was performed by aligning (without gaps) all the reads obtained for each strain against each of the 43 spacer sequences (26-bp oligos) from the direct repeats (DR) regions [38]. The number of matching reads for each spacer was counted, considering both forward and reverse-complement sequences, and accepting up to 1 nucleotide mismatch. Spacers with 0 matches were interpreted as missing.

Whole-genome alignments of the Beijing sequences to other mycobacterial strains were generated using MUMMER version 3.20 [39] to identify maximal stretches of perfectly matching regions, selecting optimal order-preserving assignments of matches between the genomes based on the longest increasing subsequence algorithm [40], and then using in-house sequence-alignment tools to determine alignments of the intervening regions with mismatches and/or gaps.

The complete genome sequences for $M$. tuberculosis strains HN878, R1207 (representative of R86 cluster), and X122 (representative of R220 cluster) have been deposited in GenBank with accession numbers ADNF01000000, ADNH01000000, and ADNG01000000, respectively.

\section{Results}

\section{Whole-Genome Sequencing}

In order to establish a complete genome sequence to use as a reference for sequencing of other members of the Beijing strain family, HN878 was chosen as a representative strain and the genome was sequenced using Solexa sequencing technology. HN878 is a fully drugsusceptible member of the modern (typical) W-Beijing family which was isolated in Houston, TX as part of a TB outbreak in the 1990's, and has been used as a reference strain in multiple studies to characterize virulence and other properties of the Beijing strain $[5,7,8]$. HN878 was sequenced in paired-end mode using 36 bp reads. Mapping of reads to unique spacer sequences in the direct-repeats (DR) region confirms that HN878 has the characteristic 000000000003771 spoligotype associated with the Beijing strain family. The genome sequence of 
M. tuberculosis H37Rv (NCBI accession: NC_000962) was used as a reference sequence for comparative assembly of the HN878 genome. The mean depth of coverage was $70.8 x$, and $98.4 \%$ of the genome was covered by at least one read. The remaining uncovered regions are primarily associated with the family of PGRS genes, and are likely due to inefficient sequencing of GC-rich regions. In $\mathrm{HN} 878,91.5 \%$ of sites with zero coverage $(60,454 / 66,065)$ were located in PGRS genes.

Among 1546 SNPs relative to H37Rv, 1296 occur in protein-coding regions. Only 1063 of 3989 genes contain a polymorphism of any type, and the remaining $73 \%$ of the genes in the genome are identical with the sequence in H37Rv. 75 genes contain frameshift mutations (see Additional file 1, Table S1). No mutations typically associated with drug resistance were found in the following genes, consistent with the pan-susceptible phenotype of this clinical isolate: $i n h A, k a t G$, eth $A, \operatorname{gyr} A$, iniABC, kasA, ndh, rpoB, rpsL, rrs, pncA, and embB. HN878 has the katG R643L and gyrA S95T alleles, confirming its membership in principle genetic group 1 [4]. Further details on SNPs and indels in HN878 (Table S2) relative to H37Rv, including IS6110 transpositions (Table S3) are described in the Additional file 1.

Then the genomes of the 14 Beijing clinical isolates from South Africa were sequenced in paired-end mode, using HN878 as a reference sequence (Table 2). The coverage ranged between $29 x$ and $98 x$, and the completion was $98.5-99.4 \%$.

There was a high concordance of IS6110 insertion sites between R220 strains and HN878 (see Figure 2). Of the 21 insertion sites in HN878, four are absent in R220, although an additional four novel insertion sites are present in this cluster (see Additional file 1, Table S4). Similar to HN878, R220 has a single insertion in the NTF region, grouping it with HN878 in the modern sublineage of Beijing strains [34]. The R220 strains lack the RD150 deletion characteristic of sublineage 7 [33,41], having Rv1671-Rv1674 intact and placing them in sublineage 6 . The six R86 strains were all found to have the Beijing spoligotype and a common set of 14 IS6110 insertion sites (Additional file 1, Table S5), including one insertion in the dnaA-dnaN region. However, only 6 of these sites were shared with HN878. The R86 strains do not have any insertion in the NTF region ( 3.48 Mb), classifying them as "ancestral" or "atypical" Beijing lineages [34].

Table 2 shows that the number of SNPs and indels was lower between the R220 strains and HN878 compared to the R86 strains, suggesting they are more closely related to HN878. The number of SNPs and indels compared to HN878 were 665-799 and 49-61, respectively, for the cluster R86 strains, and 267-392 and 35-54, respectively, for the cluster R220 strains.
Table 2 Sequencing statistics on Beijing genotype strains from South Africa

\begin{tabular}{llllll}
\hline strain ID & Cluster & completion & coverage & SNPs* & indels* \\
\hline R1207 & R86 & $98.77 \%$ & $66.9 x$ & 689 & 58 \\
X132 & R86 & $98.71 \%$ & $40.3 x$ & 671 & 61 \\
X28 & R86 & $98.35 \%$ & $57.6 x$ & 681 & 51 \\
R1746 & R86 & $98.26 \%$ & $50.8 x$ & 665 & 49 \\
X156 & R86 & $98.69 \%$ & $106.4 x$ & 799 & 57 \\
X85 & R86 & $98.78 \%$ & $97.7 x$ & 714 & 60 \\
R1909 & R220 & $98.94 \%$ & $32.8 x$ & 297 & 54 \\
R1842 & R220 & $99.15 \%$ & $58.2 x$ & 324 & 49 \\
X122 & R220 & $98.60 \%$ & $64.1 \times$ & 299 & 41 \\
R1390 & R220 & $98.50 \%$ & $31.3 x$ & 273 & 44 \\
X189 & R220 & $98.85 \%$ & $87.6 x$ & 392 & 42 \\
R1505 & R220 & $98.55 \%$ & $29.0 x$ & 267 & 39 \\
R1441 & R220 & $99.03 \%$ & $82.7 x$ & 295 & 35 \\
X29 & R220 & $99.17 \%$ & $82.0 x$ & 315 & 41 \\
\hline
\end{tabular}

*The number of SNPs and indels are assessed relative to HN878.

The clinical isolates in the R220 cluster are fairly homogeneous at the genomic level. Among 1573 polymorphic sites between the R220 strains and H37Rv (SNPs not involving PPE genes, PGRS genes, or repetitive elements), 1234 differences $(78.4 \%)$ were shared among HN878 and all 8 R220 strains. Some of these differences from $\mathrm{H} 37 \mathrm{Rv}$ might be shared with other strain families like LAM. There were 113 sites (7.2\%) where the R220 strains shared a difference from H37Rv but HN878 did not, and there were 91 sites (5.8\%) where HN878 differed from H37Rv but the R220 strains did not. Each of the strains except one had a small number of unique SNPs (1-12).

R86 strains are found to have a set of SNPs compared to H37Rv that only partially overlaps with the SNPs in HN878. Out of a selected set of 1885 SNPs, 1126 (59.7\%) were found to be common among HN878 and all six R86 strains relative to H37Rv, HN878 has 297 (15.8\%) unique SNPs, and the R86 strains have 256 (13.6\%) shared SNPs not in HN878. Thus, while both

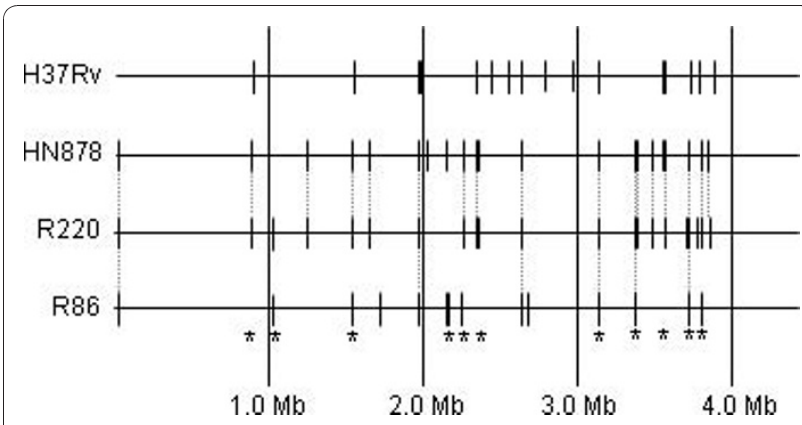

Figure 2 Positions of IS6110 insertion sites. Dashed lines indicate identical sites. Asterisks indicate sites where there are two or more close but distinct insertions separated by $27-663$ bp. 
HN878 and the R86 share many polymorphisms presumably acquired in a common ancestor of the Beijing family, they have each diverged significantly, reflecting the difference in their lineages (sublineage 1 for R86 versus sublineage 6 for HN878).

When SNPs at synonymous sites are categorized into specific base-pair substitutions, the majority of replacements $(\sim 60 \%)$ are found to be G:C to A:T transversions (68/112 in the R86 cluster, and 75/126 in the R220 cluster) (Figure 3). However, a similar bias is observed among synonymous SNPs in CDC1551 or F11 (186/374 combined) when compared to $\mathrm{H} 37 \mathrm{Rv}\left(\chi^{2}=7.36<11.07\right.$ for the distribution of SNPs in the R220 cluster versus those in CDC1551/F11, which means the difference in substitution patterns is not significant at the $p=0.05$ confidence level, $d f=5$ ), suggesting that the effect is not due to a Beijing-specific shift in the types of mutation.

\section{Drug-Resistance Mutations}

Among the Beijing clinical isolates from South Africa, well-known polymorphisms are observed that correlate with drug resistance patterns. Drug-resistance polymorphisms for the 14 clinical isolates are shown in Table 3. All of the Beijing isolates from the Western Cape, South Africa included in this study are isoniazidresistant, whereas HN878 is susceptible. All six R86 strains have the $\mathrm{S} 315 \mathrm{~T}$ mutation in katG, explaining the isoniazid resistance, and suggesting it was acquired prior to divergence of this cluster. X156 and X85 also have the c-15t inhA promoter mutation, and R1207, X132, and $\mathrm{X} 28$ have $\mathrm{c}-17 \mathrm{t}$ in the inhA promoter. inhA promoter mutations are generally considered to confer a lower-level of resistance to isoniazid (2-4 fold) as well as high-level cross-resistance to ethionamide [42]. All 6 strains also have the mutation A381P in eth $A$, a

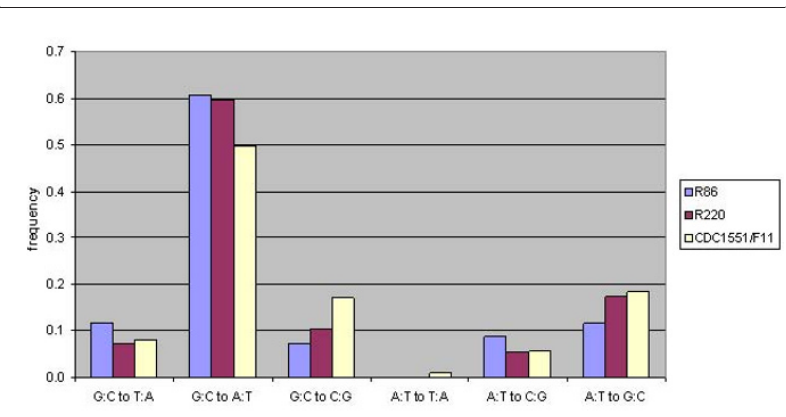

Figure 3 Substitutions at synonymous sites categorized by specific base-pair replacements, showing a similar preference for $\mathrm{G}: \mathrm{C}$ to $\mathrm{A}: \mathrm{T}$ transversions among isolates of the R86 cluster and R220 cluster as for other mycobacterial strains such as CDC1551 and F11. All substitutions were based on comparison to H37Rv. The total number of synonymous SNPs analyzed was 112, 126, and 537 for R86, R220, and CDC1551/F11, respectively. prodrug-activator, which has been suggested to also confer high-level resistance to ethionamide [43]. The polymorphisms in the R220 genome sequences are also largely consistent with the reported drug-susceptibility profiles, with the exception of ethionamide sensitivity in isolates X122, X189, and X29 (Table 4). This may relate to anomalies in drug susceptibility testing, as all R220 isolates have the c-15t inhA promoter mutation, which should cause ethionamide resistance [43]. In contrast to the cluster R86 isolates, the R220 isolates show no mutations in katG. Strains R1909 and X189 also have mutation Ile194Thr in the coding region of inhA, which occurs in the active site of the enoyl-ACP reductase and interferes with binding of INH-NAD and ETH-NAD adducts $[44,45]$.

Rifampicin resistance is explained in three R86 strains (R1207, X132, and X28) by the D435V mutation in rpoB, while the other three R86 strains (R1746, X156, and X85) have S450L (equivalent to amino acids 516 and 531 in the conventional rpoB numbering based on E. coli). Both mutations are known to confer resistance to rifampicin [46]. S450L (TCG->TTG) is the most frequently observed mutation in cultures selected on rifampicin in vitro, implying lowest fitness cost [32]. D435V (GAC->GTC) is much less common in vitro ( 1\%) [47], but is frequently observed clinically $[46,47]$. Four of the five R220 isolates that are rifampicin-resistant (R1909, $\mathrm{X} 122$, X189, and R1505) have a mutation in rpoB (either S450L or H445Y). However, isolate X29 (rifampicinresistant) does not have any mutations in $r p o B$, and conversely, isolate R1842 (rifampicin-sensitive) has S450L in rpoB, which in turn may reflect laboratory error. The H445Y mutation is less frequent among clinical isolates, but also occurs in the rifampicin binding site in RNA polymerase.

Resistance to aminoglycosides can result from a variety of polymorphisms, generally related to the small subunit of the ribosome. Mutations that confer resistance to streptomycin and similar compounds are usually found in the 500-bp or 900-bp region of rrs, the $16 \mathrm{~S}$ rRNA, or in rpsL, one of the ribosomal proteins [48]. All six R86 strains have the a514c mutation in rrs, conferring streptomycin resistance. X28 and X156 showed streptomycin resistance in drug-susceptibility testing; data on the other four strains was not available. Kanamycin binds a different site on the ribosome and shows cross-resistance with amikacin and capreomycin [49]. The most common mutation associated with kanamycin resistance is a1401g in rrs [50]. All R86 strains except isolate R1746 have the a1401g mutation in rrs. Four of the six strains were kanamycin-resistant (the other two were not tested). The capreomycin sensitivity of isolate X85 is inconsistent with the kanamycin/amikacin resistance, and might be an anomaly in drug- 
Table 3 Drug resistance mutations among $\mathbf{R} 86$ cluster isolates.

\begin{tabular}{lllllllllll}
\hline strain & Resist. & sens. & katG & inhA & rpoB & embB & pncA & rrs & ethA & gyrA \\
\hline R1207 & IR & B & S315T & g-17t & D435V & M306l & + C L172 & a514C, a1401g & A381P & D94H \\
X132 & IRACSK & EOB & S315T & g-17t & D435V & M306l & + C L172 & a514C, a1401g & A381P & wt \\
X28 & IRACOSK & EB & S315T & g-17t & D435V & M306l & + C L172 & a514C, a1401g & A381P & D94N \\
R1746 & IR & B & S315T & wt & S450L & M306l & C14R & a514C & A381P & wt \\
X156 & IRACSK & EOB & S315T & C-15t & S450L & M306l & C14R & a514C, a1401g & A381P & wt \\
X85 & IRAOKE & CB & S315T & C-15t & S450L & M306l & C14R & a514C, a1401g & A381P & D94G \\
\hline
\end{tabular}

wt $=$ wild-type, $\mathrm{I}=$ isoniazid, $\mathrm{R}=$ rifampicin, $\mathrm{S}=$ streptomycin, $\mathrm{A}=$ amikacin, $\mathrm{C}=$ capreomycin, $\mathrm{K}=$ kanamycin, $\mathrm{E}=$ ethionamide, $\mathrm{B}=$ ethambutol, $\mathrm{O}=$ ofloxacin.

susceptibility testing. In the R220 cluster, isolates X189 and X29 are resistant to kanamycin and amikacin, and both strains show the expected a1401g mutation in $\mathrm{rrs}$, whereas X122 (amikacin-sensitive) does not. R1842 has rrs c517t and X122 has K43R in ribosomal protein rpsL, each of which should confer streptomycin resistance, though this was not tested.

Resistance to fluoroquinolones is typically caused by mutations in gyrA [51]. Isolates X28 and X85 were reported to be ofloxacin-resistant, and X132 and X156 were reported to be ofloxacin-sensitive (data for R1207 and R1746 was not available). Three different amino acid mutations were observed in gyrA among these six strains: D94H in isolate R1207, D94N in X28, and D94G in X85. The SNPs in isolates X28 and X85 explain their ofloxacin resistance, and the fact that X132 and X156 have wild-type gyrA sequences is consistent with their ofloxacin sensitivity. The ofloxacin resistance of isolates $\mathrm{X} 122$ and X189 can be explained by mutations in D94 in $g y r A$. Isolate $\mathrm{X} 29$, which is sensitive to oflaxacin, has a wild-type gyrA sequence.

All six R86 strains have the mutation M306I in $e m b B$, commonly associated with resistance to ethambutol [52], although all six strains were reported to be sensitive to ethambutol by drug-susceptibility testing. It is well known that drug-susceptibility testing underreports ethambutol resistance [53]. Note that, while all 6 strains have a mutation from Met to Ile, they use different codons; 'ATA' (for R1207, X132, and X28) and 'ATC' (for R1746, X156, and X85). Many of the R220 strains have a mutation in Met306 in $e m b B$, either to
Val or Ile, although most of the strains tested sensitive to ethambutol as well.

Although resistance to pyrazinamide was not tested, 10 of the 14 strains showed mutations in $p n c A$ (pyrazinamidase). One R86 isolate (X29) has a frameshift mutation of $8 \mathrm{bp}$ in $p n c A$, while another (X189) had a large deletion of 881 bp knocking out the entire coding region, along with the adjacent gene, Rv2044c. Strain R1909 has mutation D8N, and strain X122 has Y103* (truncation mutation). All six R220 strains show mutations in $p n c A$ : three strains have a frameshift mutation (+c in codon 172), and three have amino acid substitution (C14R). Multiple mutations, including frameshifts, throughout $p n c A$ are associated with resistance to pyrazinamide [54-56].

\section{Non-clonal Acquisition of Drug-Resistance Mutations}

In order to better understand the origins of drug resistance among the Beijing strains, a phylogenetic tree was constructed to identify their evolutionary relationships. Subsequently, drug resistance mutations were mapped onto the tree to determine whether the patterns of drug resistance (associations between strains) could be explained by the same topology (i.e. inheritance). A master set of 727 polymorphic sites (SNPs only) with good depth of coverage $(\geq 10 x$, i.e. sites covered by at least 10 reads) across all sequenced strains was selected. A subset of 704 sites was produced by removing those involved in drug resistance (e.g. $\operatorname{inh} A$, katG, gyrA, rpoB, $p n c A, r r s, e m b B)$. The 704 sites were used to construct a maximum parsimony tree using dnapars in PHYLIP

Table 4 Drug resistance mutations among R220 cluster isolates

\begin{tabular}{|c|c|c|c|c|c|c|c|c|c|c|}
\hline strain & resist. & sens. & katG & $\operatorname{inh} A$ & $r p o B$ & $e m b B$ & pncA & $r r s$ & $r p s L$ & gyrA \\
\hline R1909 & IRB & & wt & $c-15 t$, I194T & S450L & M306V & D8N & a1401g & wt & wt \\
\hline R1842 & 1 & $\mathrm{RB}$ & wt & $c-15 t$ & S450L & M306V & wt & $c 517 t$ & wt & D94A \\
\hline$\times 122$ & $\mathrm{IRO}$ & $E A B$ & wt & $c-15 t$ & S450L & M306I & Y103* & wt & K43R & D94G \\
\hline R1390 & 1 & & wt & $c-15 t$ & wt & wt & wt & wt & wt & wt \\
\hline X189 & IRACOK & ES & wt & $c-15 t, 1194 T$ & S450L & M306V & 881 bp del & a1401g & wt & D94A \\
\hline R1505 & $\mathbb{R}$ & $B$ & wt & $c-15 t$ & H445Y & wt & wt & wt & wt & wt \\
\hline R1441 & I & $\mathrm{RB}$ & wt & $c-15 t$ & wt & M306I & wt & wt & wt & wt \\
\hline$\times 29$ & IRASK & BOCE & wt & $c-15 t$ & wt & M306V & 8 bp del & a1401g & wt & wt \\
\hline
\end{tabular}


3.66 [57]. Figure 4 shows the phylogenetic relationship with representative branch lengths, clearly showing the tight clustering of the two groups, cluster R86 (X85, X156, R1746, X132, X28, R1207) and the R220 cluster (R1441, R1505, R1390, X29, R1842, X189, R1909, X122), indicating that both groups have evolved independently from a common ancestor.

The number of SNPs differentiating the strains within each cluster is small but non-zero. Since the relationships are difficult to see in Figure 4a, the phylogeny was re-drawn as a cladogram (without meaningful branch lengths) in Figure 4b, and each branch is labeled with the number of SNPs associated with it. The observation that cluster R86 isolates share 236 SNPs while the R220 isolates share 303 unique SNPs suggests that these two clusters are largely clonal, and that isolates within the clusters are differentiated from one another by a limited number of SNPs. For example, the similarity between X29 and R1842 is supported by two unique SNPs: Q219P in Rv2571c and V16V in proW. The SNPs associated with each branch point are listed in Additional file 1, Table S7. It is also interesting to note that some individual isolates have continued to diverge, for example isolate R1746 has accumulated 12 unique polymorphisms. These differentiating SNPs likely represent recent evolutionary events. According to this phylogeny, HN878 is more closely related to the R220 cluster (sharing 195 SNPs, compared to 0 shared with the R86 cluster, using H37Rv as the outgroup), but is distinguished from the R220 members by a further 195 unique differences $(108+87)$. It is important to note that these polymorphisms do not necessarily imply anything about the overall population structure, due to the non-random nature of the sample. Nonetheless, they represent the diversity and inter-relationships among the 14 clinical isolates sequenced.

When mutations related to drug resistance were projected onto this phylogeny, the patterns were frequently found to disagree with the phylogeny above. The most parsimonious explanation for this observation is that mutations conferring resistance to a given drug have arisen multiple times independently within both the R220 and R86 clusters. Figure 5 shows the pattern of mutations in gyrA conferring fluoroquinolone resistance. In these 14 Beijing strains, mutations in Asp94 appear to have arisen 6 times independently, each associated with a unique isolate (i.e. they were not clustered in the cladogram). In fact, Asp94 is mutated to 4 different amino acids (Gly: X85, X122, Asn: X28, His: R1207, Ala:

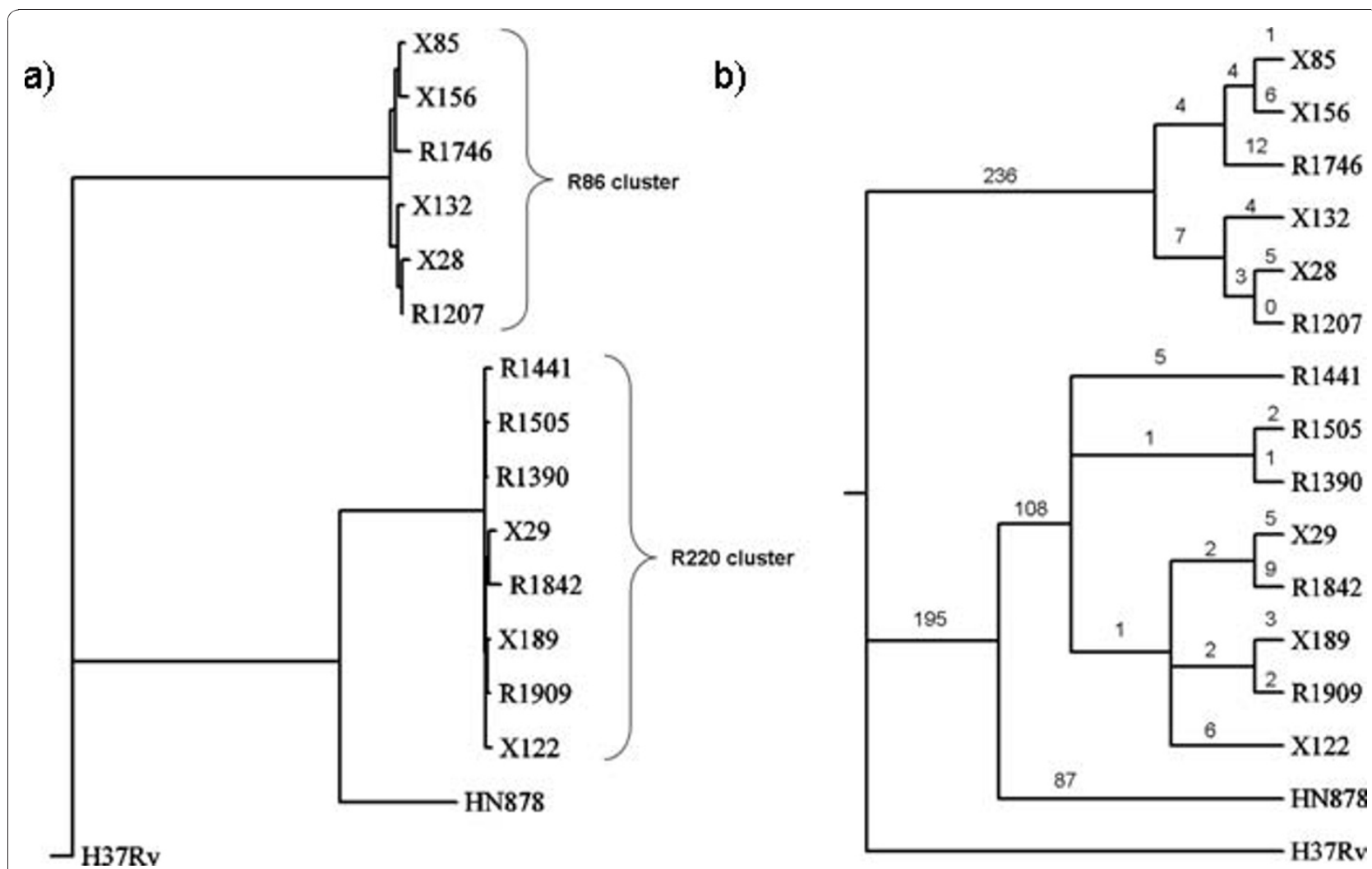

Figure 4 Phylogenetic tree constructed from 727 SNPs (excluding those related to drug resistance) by maximum parsimony (a), and also displayed as a cladogram (b) showing the number of changes (unique SNPs) associated with each branch. 


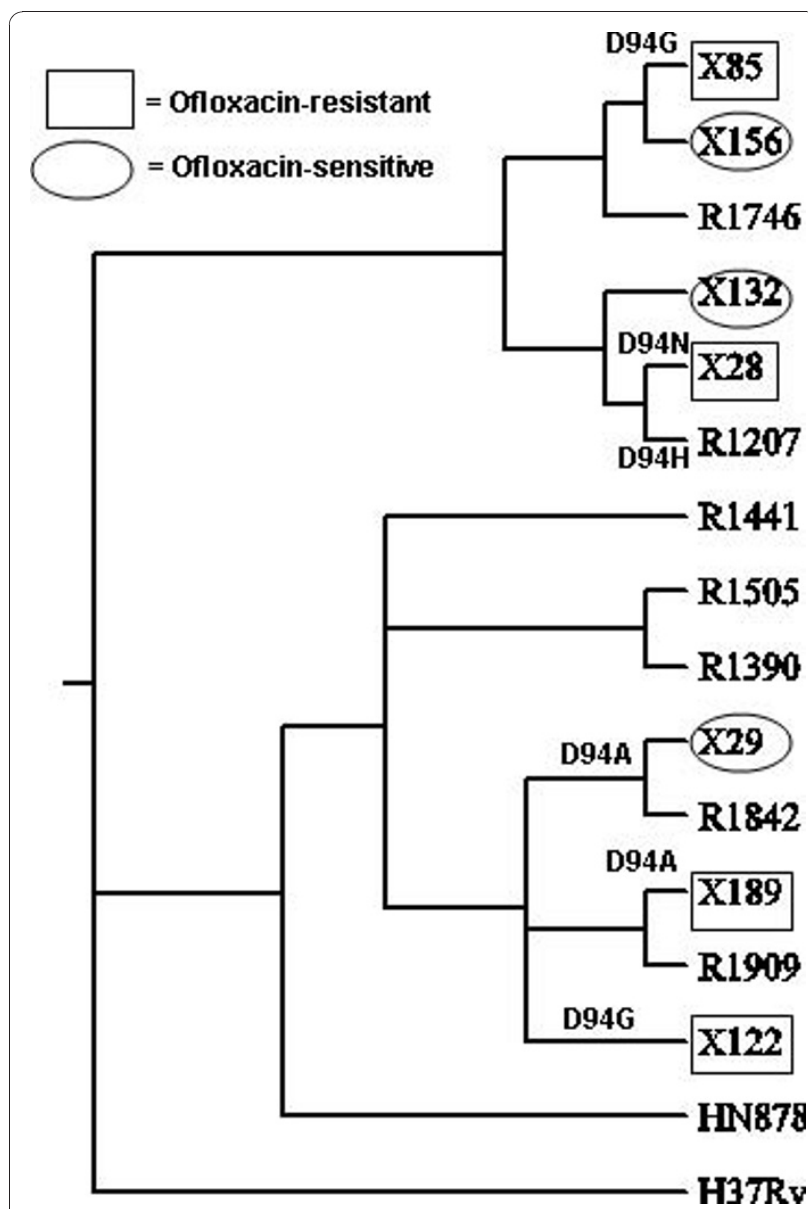

Figure 5 Mutations in gyrA related to fluoroquinolone resistance. Ofloxacin-resistant strains are boxed; Ofl-sensitive strains are encircled; other strains were not tested by DST.

X29, R1842, X189), again supporting the uniqueness of these events.

Mutations in $r p o B$ related to rifampicin resistance are shown in Figure 6. In the R86 cluster, the six isolates divide into two groups: three isolates with the S450L mutation, and three with the D435V mutation. Similarly, the $\mathrm{S} 450 \mathrm{~L}$ mutation also explains the rifampicin resistance in a group of five isolates of the R220 cluster, clearly demonstrating that each cluster has acquired rifampicin resistance independently. It is important to note that three of the eight R220 isolates did not bear the S450L mutation. Although many R220 isolates have the S450L polymorphism [58], as of 2006 only $73 \%$ of R220 isolates were RIF-resistant [27], indicating that this polymorphism had not yet achieved fixation in the population, and thus the population structure has not yet been taken over by a distinct MDR clone. Of the three R220 isolates without the S450L mutation, two had wild-type $r p o B$ sequences and were RIF-susceptible,

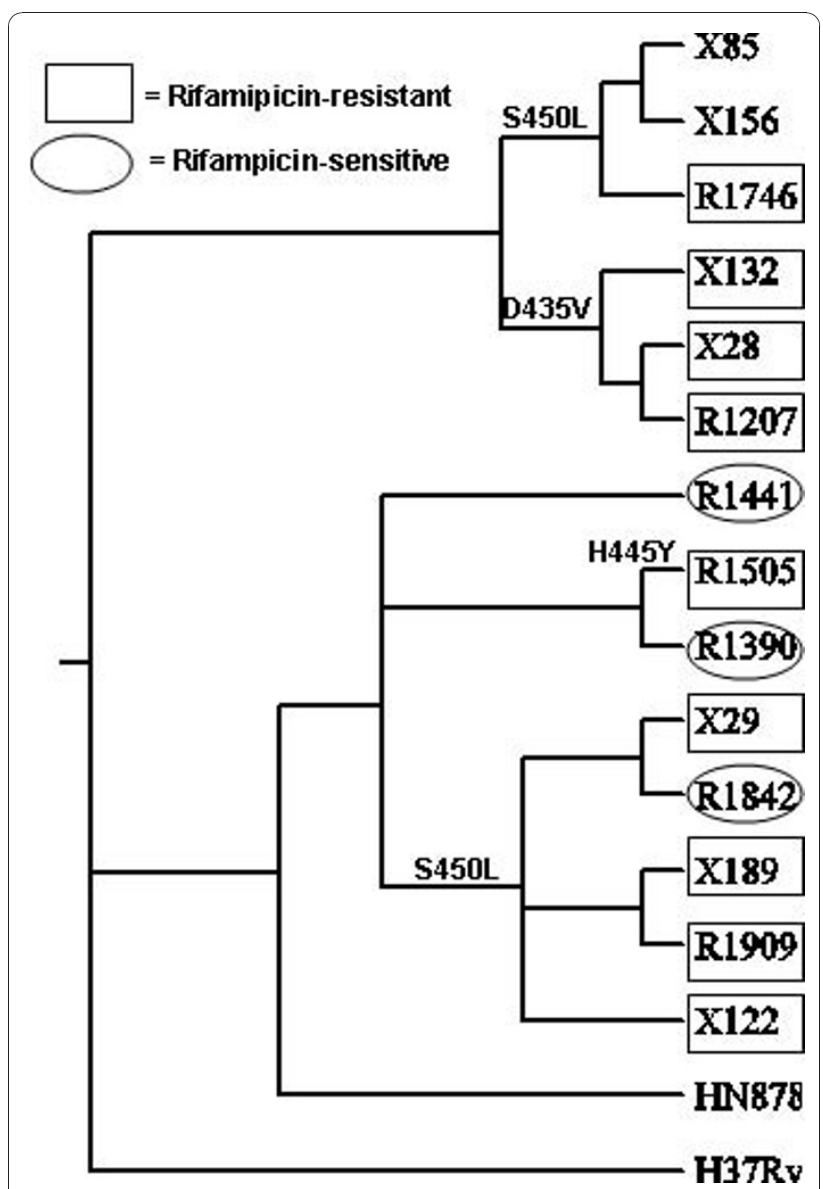

Figure 6 Mutations in rpoB related to rifampicin resistance.

and the third had a distinct RIF-resistance mutation: $\mathrm{H} 445 \mathrm{Y}$.

With respect to isoniazid resistance, Figure 7 shows that the $\mathrm{c}-15 \mathrm{t}$ inhA promoter mutation arose independently in both the R220 and R86 clusters. Whereas the c-15t mutation is found in all 8 strains of R220, it is only found in 2 of the 6 strains in the R85 cluster. However, the g-17t promoter mutation occurs in three other strains in the R86 cluster. The c-15t and g-17t inhA promoter mutations in the R86 cluster both occurred in the context of the katG S315T mutation, which occurs on an earlier branch and is found in all the strains of the R86 cluster. Simultaneous mutations in katG and the $i n h A$ promoter are frequently observed in clinical isolates [42], although it is unexpected that the $\operatorname{inh} A$ promoter mutations, which confer lower-level resistance to isoniazid, apparently occurred second. In contrast, the Ile194Thr mutation in inhA in isolates X189 and R1909 appears to have been acquired subsequent to the c-15t inhA promoter mutation common to the R220 strains, probably conferring higher-level resistance. 


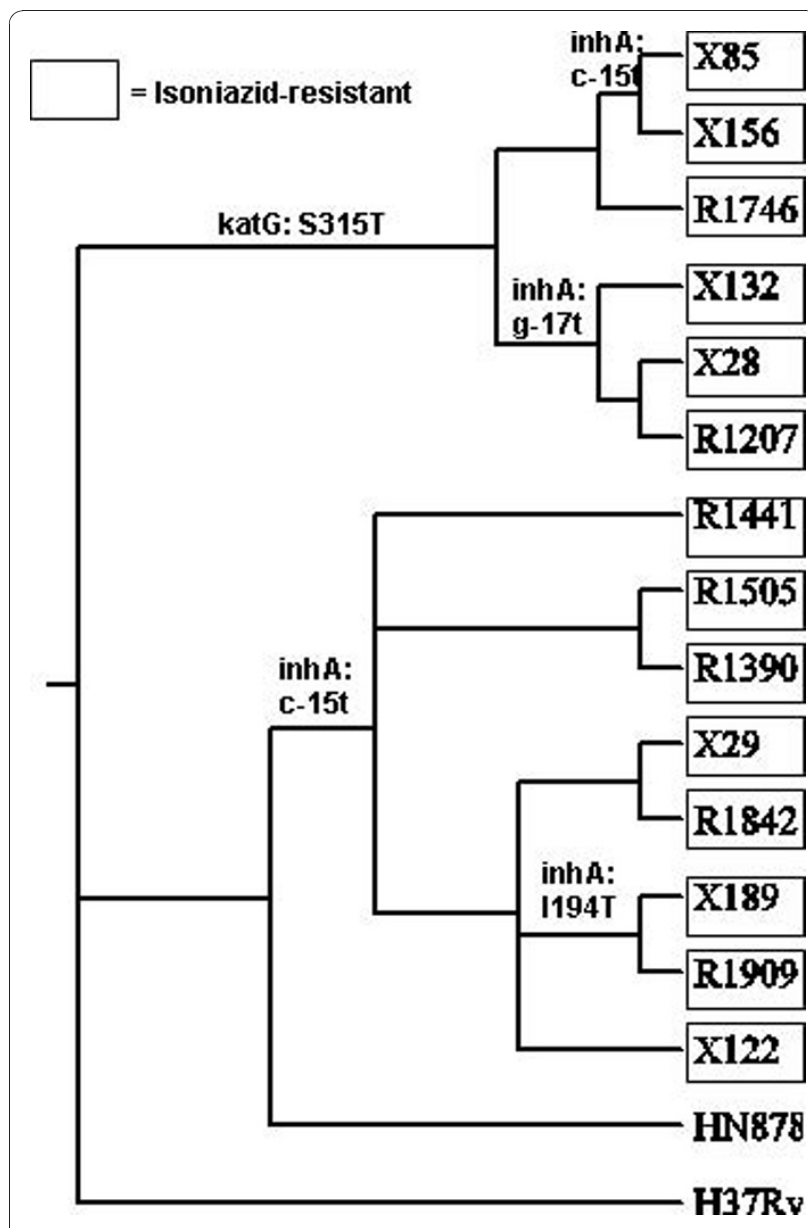

Figure 7 Mutations in inhA and katG related to isoniazid resistance. All strains except HN878 are isoniazid-resistant.

When the mutations in rrs and rpsL are mapped onto the phylogeny of the Beijing isolates (Figure 8), it is found that the R86 strains all shared mutation a516g in rrs, suggesting that streptomycin resistance arose in a common ancestor. The rrs a1401g mutation, responsible for kanamycin resistance, was observed in 5 of the 6 cluster R86 isolates, but not in isolate R1746. Therefore, it most likely has arisen twice within this cluster (unless there was a reversion to wild-type in R1746). In the R220 cluster, mutations (and most likely aminoglycoside resistance) were less prevalent. Isolate R1842 has a unique c517t rrs mutation that presumably also causes streptomycin resistance, and X122 is the only strain that has the K43R mutation in rpsL.

Finally, among the 14 Beijing isolates (both clusters), there appear to be six distinct mutations of Met306 in $e m b B$ (Figure 9).

\section{Discussion}

Our data suggests that extensive drug-resistance (beyond MDR) among Beijing strains in the Western

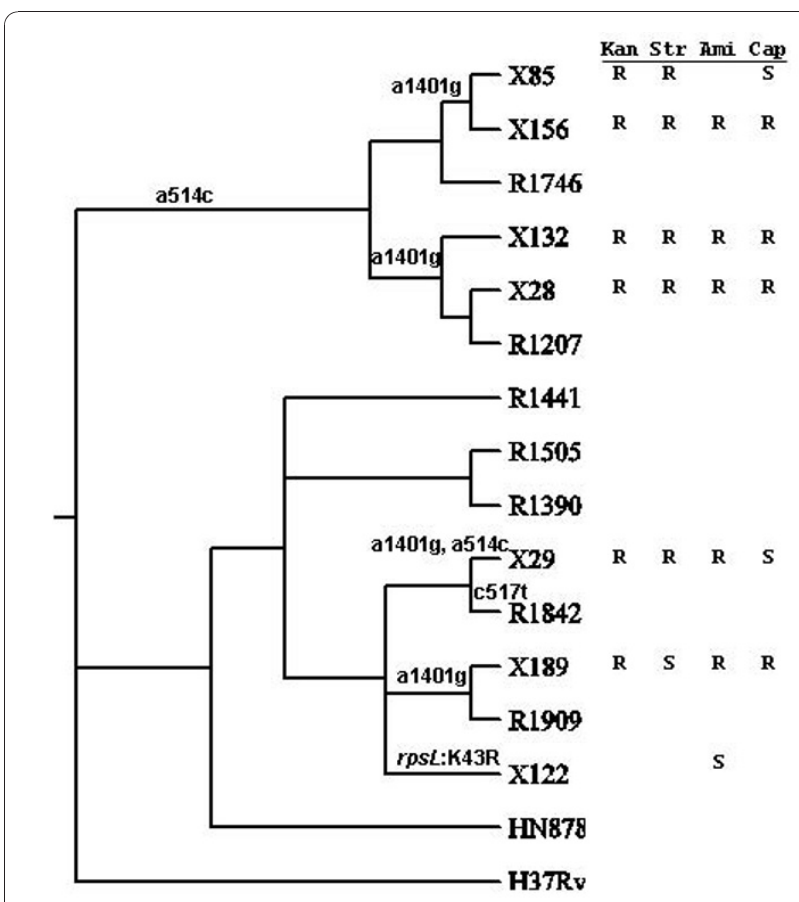

Figure 8 Mutations in $r r s$ and $r p s L$ related to aminoglycoside resistance. Kan = kanamycin, $\mathrm{Ami}=$ amikacin, $\mathrm{Cap}=$ capreomycin, $\mathrm{Str}=$ streptomycin. $\mathrm{R}=$ resistant. $\mathrm{S}=$ sensitive. Blank means not tested.

Cape of South Africa is evolving and spreading adaptively rather than by clonal expansion. The phylogenetic analysis suggests that MDR drug resistance is being transmitted clonally, as demonstrated by the observation that isolates in the R220 cluster share the same c-15t inhA promoter mutation, and isolates in the R86 cluster share the same KatG S315T mutation. Similarly, many of the strains share the RpoB S450L mutation (though acquired separately in each cluster). This is consistent with the view that MDR-TB in the Western Cape is spreading by transmission [27]. However, mutations in other drug-resistance-related genes are highly homoplaseous, showing that the XDR mutations are not clonally related, even within these tightly defined clusters. For example, among the eight R220 strains sequenced, fluoroquinolone resistance was acquired three times independently, ethambutol resistance was acquired four times independently, and resistance to aminoglycosides was acquired four times independently.

The repeated acquisition of drug resistance mutations in these strains suggests that XDR isolates are less fit and do not propagate or compete well among the circulating population of Beijing variants. If there were one well-adapted XDR clone that had perhaps acquired compensatory mutations, then it would presumably result in identical drug-resistance mutations being 


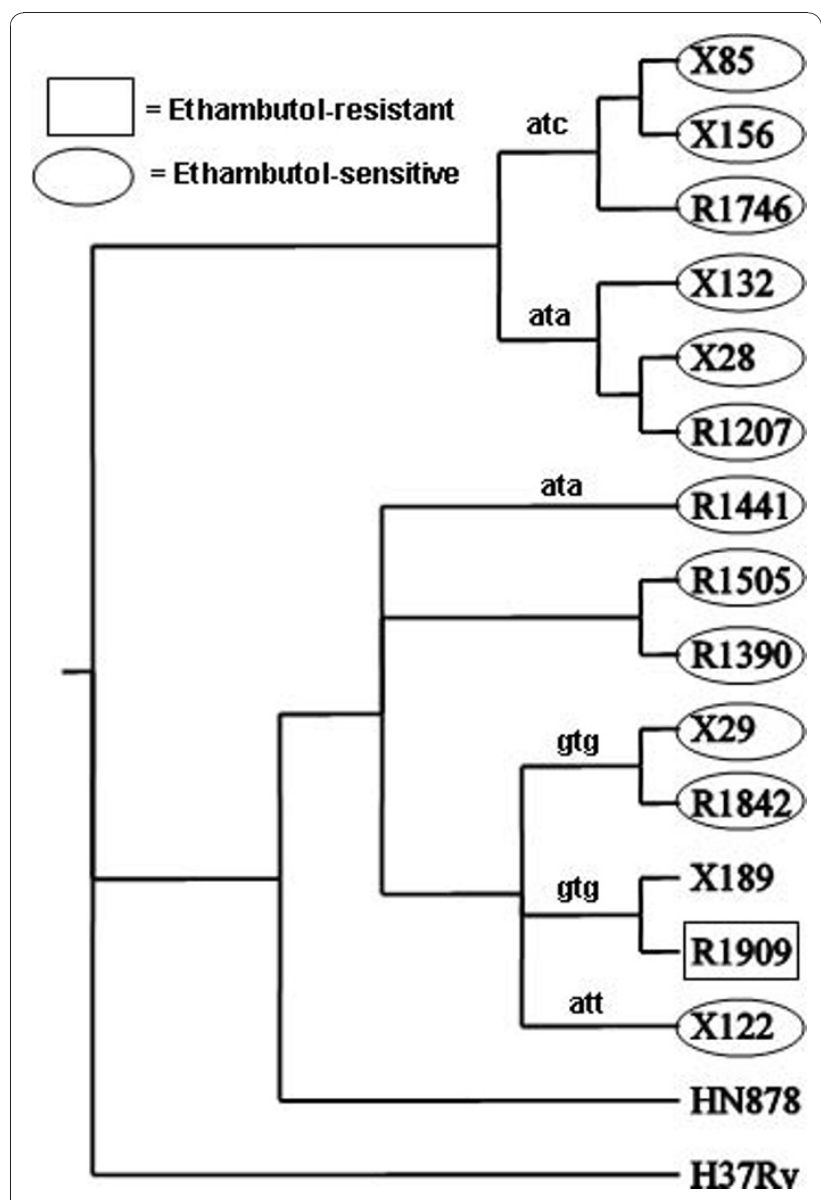

Figure 9 Mutations in $e m b B$ related to ethambutol resistance. The mutations are shown as codons replacing Met306 (atg).

transmitted through the population, which is not the case. This is consistent with the findings of van der Spuy [28], who found that, although Beijing strains as a whole were more prevalent and hence presumably more fit than other TB genotypes, drug resistant strains were not the predominant contributor to the rise of Beijing in the Cape Town region (but rather drug-susceptible isolates), probably due to increased fitness cost associated with drug-resistance mutations. Thus, although the Beijing strain as a whole appears to be more transmissible than other strains of $\mathrm{TB}$, accounting for its success and world-wide dissemination, this does not appear to directly explain the spread of XDR drug-resistance among Beijing strains, as isolates in the region display varying drug-resistance mutations and hence do not represent the transmission of an identical clone. Diversity of resistance mutations among Beijing strains has also been observed in Japan [25] and Russia [22], again indicating independent acquisition.

This pattern mimics what has been observed for the LCC strains ('Low Copy Clade,' so-called because they have only 5 copies of the IS6110 insertion sequence), which are also prevalent in South Africa [59], although LCC strains are not of the Beijing genotype, but instead are in principle genetic group 2. Cluster analysis of drug resistance mutations in LCC isolates showed that they could not all be explained by a single sequence of acquisition, suggesting they arose multiple times independently [60]. However, previous studies have examined phylogenetic relationships using only a limited set of markers, including the drug-resistance mutations themselves, whereas our study is the first to use a genomewide catalog of SNPs to assess relationships among strains independent of the drug-resistance mutations.

The repeated development of XDR drug resistance among Beijing strains of tuberculosis in the Western Cape region differs from other outbreaks of drugresistant TB, such as in New York City in the 1990's $[17,18]$, as well as in KwaZulu-Natal, South Africa more recently $[61,62]$. Each outbreak occurred in a defined geographical setting and was demonstrated to be due to transmission of clones with identical drug-resistance markers. In contrast, our findings suggest that XDR drug resistance in the Beijing strains in the Western Cape is not spreading clonally, but continues to be acquired independently in different strains. The drugresistant TB outbreak in the Western Cape of South Africa covers a wider geographical region (spanning hundreds of miles). Thus the strains we analyzed are more distantly related, although they share a common ancestor, which is in turn reflected by the number of SNPs differentiating the respective isolates. The wide distribution of these strains demonstrates how failure to contain an initial drug resistant strain leads to both spread and acquisition of additional resistance markers under the right conditions, thereby emphasizing the need for rapid and accurate diagnosis of drug resistance.

There are a number of possible explanations for the increased association of drug resistance with the Beijing strain $[34,63]$. One hypothesis is that the Beijing strain is more adaptive, allowing it to acquire mutations more rapidly, which are then selected through the application of chemotherapy. It has been hypothesized that the apparent adaptiveness of the Beijing strain could be due to mutations in DNA repair genes mutT2 and mutT4 which might produce a hypermutator phenotype [64]. However, these mutations appear only in the most recent lineages, and are not found in the more ancestral lineages, including lineage 1, of which cluster R86 is a member. So it could not explain the extensive homoplasy of drug resistance among all these isolates. Furthermore, a comparison of the types of substitutions in the genomes of R220 isolates versus R86 isolates shows that the profiles are nearly identical (Figure 3), suggesting that there have not been functional changes 
to specific DNA repair genes or mechanisms, which might have been reflected in a bias of the substitution patterns $[65,66]$. No evidence has yet been found for hypermutation among clinical isolates, and the in vitro rро $B$ mutation rate $\left(1.1 \times 10^{-8}\right)$ has been found to be in line with non-Beijing strains [67].

The primary limitation of our study is related to the selection of clinical isolates for sequencing. While all were of the Beijing genotype, the strains chosen for sequencing were selected to span a range of drug resistance from mono-resistant (all were resistant to INH) to XDR. While no sampling bias (in terms of age, gender, etc.) was intentionally applied, and the samples were isolated from patients in a variety of hospitals throughout the Western Cape region, it could be the case that our conclusions are specific to the small number of strains sequenced, or their shared resistance to isoniazid, and might not generalize to the broader TB epidemic within the region, or to outbreaks of drug-resistance and/or the Beijing strain of $M$. tuberculosis in other geographic locations around the world. Furthermore, no drugsusceptible strains from the Western Cape were sequenced, making the evolutionary relationships to the broader population of strains endemic to the region speculative. Finally, incidence of HIV is high the Western Cape region, and this could be an additional complicating factor that could influence the acquisition of drug resistance in these strains, as suggested in [68], but our study did not control for HIV status of the patients.

\section{Conclusions}

Whole-genome sequencing and phylogenetic analysis of genetic differences among clinical isolates of the Beijing genotype from the Western Cape of South Africa suggests that, while MDR-TB is spreading by transmission in the region, additional drug resistance mutations are being acquired independently, and hence the spread of extensive drug resistance (XDR) appears to be non-clonal. The successful dissemination of these drug-resistant Beijing genotypes in South Africa should be a major concern for the National Tuberculosis Control Program, as the current strategy is unable to curb the spread of these strains [27]. Failure to contain (diagnose and treat) these strains has led to the evolution of XDR-TB through further acquisition of resistance markers.

\section{Additional material}

Additional file 1: Supplementary details and comparative analysis of genome sequences of $M$. tuberculosis Beijing strains HN878, R1207 and X122. This file contains a description of the polymorphisms observed among the genome sequences of $M$. tuberculosis Beijing strains HN878, R1207 and X122, including a catalog of SNPs, frameshift mutations, and coordinates of large-scale indels and IS6110 insertion sites.

\section{Acknowledgements}

This work was support in part by funding from the Robert A. Welch Foundation, grant number A-0015 (JCS).

\section{Author details}

${ }^{1}$ Department of Computer Science and Engineering, Texas A\&M University, College Station, TX USA. ${ }^{2}$ Department of Biochemistry and Biophysics, Texas A\&M University, College Station, TX USA. ${ }^{3}$ Department of Microbiology, Immunology, and Pathology, Colorado State University, Fort Collins, CO USA. ${ }^{4}$ DST/NRF Centre of Excellence in Biomedical Tuberculosis Research, US/MRC Centre for Molecular and Cellular Biology, Division of Molecular Biology and Human Genetics, Department of Biomedical Sciences, Faculty of Health Sciences, Stellenbosch University, P.O. Box 19063, Tygerberg 7505, South Africa.

\section{Authors' contributions}

$J \mathrm{~S}$ and $\mathrm{PVH}$ conceived of and designed the experiment. KD, TV, ES, RW, and NP collected the samples, performed genotyping and drug-susceptibility testing, and provided genomic DNA. XC ran the Illumina sequencer. YF and $\mathrm{Tl}$ assembled the genome sequences and performed the analysis of the data. TI wrote the manuscript, and all authors contributed to editing it. All authors have read and approved the final manuscript.

Received: 10 July 2010 Accepted: 26 November 2010

Published: 26 November 2010

\section{References}

1. van Soolingen D, Qian L, de Haas PE, Douglas JT, Traore H, Portaels F, Qing HZ, Enkhsaikan D, Nymadawa P, van Embden JD: Predominance of a single genotype of Mycobacterium tuberculosis in countries of east Asia. J Clin Microbiol 1995, 33(12):3234-3238.

2. Bifani PJ, Mathema B, Kurepina NE, Kreiswirth BN: Global dissemination of the Mycobacterium tuberculosis W-Beijing family strains. Trends Microbiol 2002, 10(1):45-52.

3. Glynn JR, Whiteley J, Bifani PJ, Kremer K, van Soolingen D: Worldwide occurrence of Beijing/W strains of Mycobacterium tuberculosis: a systematic review. Emerg Infect Dis 2002, 8(8):843-849.

4. Sreevatsan S, Pan X, Stockbauer KE, Connell ND, Kreiswirth BN, Whittam TS, Musser JM: Restricted structural gene polymorphism in the Mycobacterium tuberculosis complex indicates evolutionarily recent global dissemination. Proc Natl Acad Sci USA 1997, 94(18):9869-9874.

5. Manca C, Tsenova L, Bergtold A, Freeman S, Tovey M, Musser JM, Barry CE, Freedman VH, Kaplan G: Virulence of a Mycobacterium tuberculosis clinical isolate in mice is determined by failure to induce Th1 type immunity and is associated with induction of IFN-alpha/beta. Proc Natl Acad Sci USA 2001, 98(10):5752-5757.

6. Lopez B, Aguilar D, Orozco H, Burger M, Espitia C, Ritacco V, Barrera L, Kremer K, Hernandez-Pando R, Huygen $K$, et al: A marked difference in pathogenesis and immune response induced by different Mycobacterium tuberculosis genotypes. Clin Exp Immunol 2003, 133(1):30-37.

7. Manca C, Tsenova L, Barry CE, Bergtold A, Freeman S, Haslett PA, Musser JM, Freedman VH, Kaplan G: Mycobacterium tuberculosis CDC1551 induces a more vigorous host response in vivo and in vitro, but is not more virulent than other clinical isolates. J Immunol 1999, 162(11):6740-6746.

8. Tsenova L, Ellison E, Harbacheuski R, Moreira AL, Kurepina N, Reed MB, Mathema B, Barry CE, Kaplan G: Virulence of selected Mycobacterium tuberculosis clinical isolates in the rabbit model of meningitis is 
dependent on phenolic glycolipid produced by the bacilli. J Infect Dis 2005, 192(1):98-106.

9. Li Q, Whalen CC, Albert JM, Larkin R, Zukowski L, Cave MD, Silver RF: Differences in rate and variability of intracellular growth of a panel of Mycobacterium tuberculosis clinical isolates within a human monocyte model. Infect Immun 2002, 70(11):6489-6493.

10. Zhang M, Gong J, Yang Z, Samten B, Cave MD, Barnes PF: Enhanced capacity of a widespread strain of Mycobacterium tuberculosis to grow in human macrophages. J Infect Dis 1999, 179(5):1213-1217.

11. Drobniewski F, Balabanova Y, Nikolayevsky V, Ruddy M, Kuznetzov S , Zakharova S, Melentyev A, Fedorin I: Drug-resistant tuberculosis, clinical virulence, and the dominance of the Beijing strain family in Russia. JAMA 2005, 293(22):2726-2731.

12. Borgdorff MW, Van Deutekom H, De Haas PE, Kremer K, Van Soolingen D: Mycobacterium tuberculosis, Beijing genotype strains not associated with radiological presentation of pulmonary tuberculosis. Tuberculosis (Edinb) 2004, 84(5):337-340.

13. Parwati I, Alisjahbana $B$, Apriani L, Soetikno RD, Ottenhoff TH, van der Zanden $A G$, van der Meer J, van Soolingen $D$, van Crevel R: Mycobacterium tuberculosis Beijing genotype is an independent risk factor for tuberculosis treatment failure in Indonesia. J Infect Dis 2010, 201(4):553-557.

14. Reed MB, Domenech P, Manca C, Su H, Barczak AK, Kreiswirth BN, Kaplan G, Barry CE: A glycolipid of hypervirulent tuberculosis strains that inhibits the innate immune response. Nature 2004, 431(7004):84-87.

15. Constant P, Perez E, Malaga W, Laneelle MA, Saurel O, Daffe M, Guilhot C: Role of the pks 15/1 gene in the biosynthesis of phenolglycolipids in the Mycobacterium tuberculosis complex. Evidence that all strains synthesize glycosylated p-hydroxybenzoic methyl esters and that strains devoid of phenolglycolipids harbor a frameshift mutation in the pks15/1 gene. J Biol Chem 2002, 277(41):38148-38158.

16. Glynn JR: Beijing/W genotype Mycobacterium tuberculosis and drug resistance. Emerg Infect Dis 2006, 12(5):736-743.

17. Frieden TR, Sherman LF, Maw KL, Fujiwara PI, Crawford JT, Nivin B, Sharp V, Hewlett D Jr, Brudney K, Alland D, et al: A multi-institutional outbreak of highly drug-resistant tuberculosis: epidemiology and clinical outcomes. JAMA 1996, 276(15):1229-1235.

18. Bifani PJ, Plikaytis BB, Kapur V, Stockbauer K, Pan X, Lutfey ML, Moghazeh SL, Eisner W, Daniel TM, Kaplan MH, et al: Origin and interstate spread of a New York City multidrug-resistant Mycobacterium tuberculosis clone family. JAMA 1996, 275(6):452-457.

19. Pfyffer GE, Strassle A, van Gorkum T, Portaels F, Rigouts L, Mathieu C, Mirzoyev F, Traore H, van Embden JD: Multidrug-resistant tuberculosis in prison inmates, Azerbaijan. Emerg Infect Dis 2001, 7(5):855-861.

20. Mlambo CK, Warren RM, Poswa X, Victor TC, Duse AG, Marais E: Genotypic diversity of extensively drug-resistant tuberculosis (XDR-TB) in South Africa. Int J Tuberc Lung Dis 2008, 12(1):99-104.

21. Almeida D, Rodrigues C, Ashavaid TF, Lalvani A, Udwadia ZF, Mehta A: High incidence of the Beijing genotype among multidrug-resistant isolates of Mycobacterium tuberculosis in a tertiary care center in Mumbai, India. Clin Infect Dis 2005, 40(6):881-886.

22. Toungoussova OS, Caugant DA, Sandven P, Mariandyshev AO, Bjune G: Impact of drug resistance on fitness of Mycobacterium tuberculosis strains of the W-Beijing genotype. FEMS Immunol Med Microbiol 2004, 42(3):281-290.

23. Park YK, Shin S, Ryu S, Cho SN, Koh WJ, Kwon OJ, Shim YS, Lew WJ, Bai GH: Comparison of drug resistance genotypes between Beijing and nonBeijing family strains of Mycobacterium tuberculosis in Korea. J Microbiol Methods 2005, 63(2):165-172.

24. Anh DD, Borgdorff MW, Van LN, Lan NT, van Gorkom T, Kremer K, van Soolingen D: Mycobacterium tuberculosis Beijing genotype emerging in Vietnam. Emerg Infect Dis 2000, 6(3):302-305.

25. Iwamoto T, Yoshida S, Suzuki K, Wada T: Population structure analysis of the Mycobacterium tuberculosis Beijing family indicates an association between certain sublineages and multidrug resistance. Antimicrob Agents Chemother 2008, 52(10):3805-3809.

26. Hillemann D, Kubica T, Rusch-Gerdes S, Niemann S: Disequilibrium in distribution of resistance mutations among Mycobacterium tuberculosis Beijing and non-Beijing strains isolated from patients in Germany. Antimicrob Agents Chemother 2005, 49(3):1229-1231.
27. Johnson R, Warren RM, van der Spuy GD, Gey van Pittius NC, Theron D, Streicher EM, Bosman M, Coetzee GJ, van Helden PD, Victor TC: Drugresistant tuberculosis epidemic in the Western Cape driven by a virulent Beijing genotype strain. Int I Tuberc Lung Dis 2010, 14(1):119-121.

28. van der Spuy GD, Kremer K, Ndabambi SL, Beyers N, Dunbar R, Marais BJ, van Helden PD, Warren RM: Changing Mycobacterium tuberculosis population highlights clade-specific pathogenic characteristics. Tuberculosis (Edinb) 2009, 89(2):120-125.

29. Streicher EM, Warren RM, Kewley C, Simpson J, Rastogi N, Sola C, van der Spuy GD, van Helden PD, Victor TC: Genotypic and phenotypic characterization of drug-resistant Mycobacterium tuberculosis isolates from rural districts of the Western Cape Province of South Africa. J Clin Microbiol 2004, 42(2):891-894.

30. Richardson M, van Lill SW, van der Spuy GD, Munch Z, Booysen CN, Beyers N, van Helden PD, Warren RM: Historic and recent events contribute to the disease dynamics of Beijing-like Mycobacterium tuberculosis isolates in a high incidence region. Int I Tuberc Lung Dis 2002, 6(11):1001-1011.

31. Warren R, Richardson M, van der Spuy G, Victor T, Sampson S, Beyers N, van Helden P: DNA fingerprinting and molecular epidemiology of tuberculosis: use and interpretation in an epidemic setting. Electrophoresis 1999, 20(8):1807-1812.

32. Gagneux S, Long CD, Small PM, Van T, Schoolnik GK, Bohannan BJ: The competitive cost of antibiotic resistance in Mycobacterium tuberculosis. Science 2006, 312(5782):1944-1946.

33. Hanekom M, van der Spuy GD, Streicher E, Ndabambi SL, McEvoy CR, Kidd M, Beyers N, Victor TC, van Helden PD, Warren RM: A recently evolved sublineage of the Mycobacterium tuberculosis Beijing strain family is associated with an increased ability to spread and cause disease. J Clin Microbiol 2007, 45(5):1483-1490.

34. Mokrousov I, Jiao WW, Sun GZ, Liu JW, Valcheva V, Li M, Narvskaya O, Shen AD: Evolution of drug resistance in different sublineages of Mycobacterium tuberculosis Beijing genotype. Antimicrob Agents Chemother 2006, 50(8):2820-2823.

35. Marais BJ, Victor TC, Hesseling AC, Barnard M, Jordaan A, Brittle W, Reuter H, Beyers N, van Helden PD, Warren RM, et al: Beijing and Haarlem genotypes are overrepresented among children with drug-resistant tuberculosis in the Western Cape Province of South Africa. J Clin Microbiol 2006, 44(10):3539-3543.

36. Middlebrook G, Cohn ML: Bacteriology of tuberculosis: laboratory methods. Am J Public Health Nations Health 1958, 48(7):844-853.

37. Larsen MH, Biermann K, Tandberg S, Hsu T, Jacobs WR Jr: Genetic Manipulation of Mycobacterium tuberculosis. Curr Protoc Microbiol 2007, Chapter 10(Unit 10A):12.

38. Kamerbeek J, Schouls L, Kolk A, van Agterveld M, van Soolingen D, Kuijper S, Bunschoten A, Molhuizen H, Shaw R, Goyal M, et al: Simultaneous detection and strain differentiation of Mycobacterium tuberculosis for diagnosis and epidemiology. J Clin Microbiol 1997, 35(4):907-914

39. Kurtz S, Phillippy A, Delcher AL, Smoot M, Shumway M, Antonescu C, Salzberg SL: Versatile and open software for comparing large genomes. Genome Biol 2004, 5(2):R12.

40. Delcher AL, Phillippy A, Carlton J, Salzberg SL: Fast algorithms for largescale genome alignment and comparison. Nucleic Acids Res 2002, 30(11):2478-2483.

41. Tsolaki AG, Gagneux S, Pym AS, Goguet de la Salmoniere YO, Kreiswirth BN, Van Soolingen D, Small PM: Genomic deletions classify the Beijing/W strains as a distinct genetic lineage of Mycobacterium tuberculosis. I Clin Microbiol 2005, 43(7):3185-3191.

42. Morlock GP, Metchock B, Sikes D, Crawford JT, Cooksey RC: ethA, inhA, and katG loci of ethionamide-resistant clinical Mycobacterium tuberculosis isolates. Antimicrob Agents Chemother 2003, 47(12):3799-3805.

43. DeBarber AE, Mdluli K, Bosman M, Bekker LG, Barry CE: Ethionamide activation and sensitivity in multidrug-resistant Mycobacterium tuberculosis. Proc Natl Acad Sci USA 2000, 97(17):9677-9682.

44. Dessen A, Quemard A, Blanchard JS, Jacobs WR Jr, Sacchettini JC: Crystal structure and function of the isoniazid target of Mycobacterium tuberculosis. Science 1995, 267(5204):1638-1641. 
45. Wang F, Langley R, Gulten G, Dover LG, Besra GS, Jacobs WR Jr, Sacchettini JC: Mechanism of thioamide drug action against tuberculosis and leprosy. J Exp Med 2007, 204(1):73-78.

46. Ramaswamy S, Musser JM: Molecular genetic basis of antimicrobial agent resistance in Mycobacterium tuberculosis: 1998 update. Tuber Lung Dis 1998, 79(1):3-29

47. Strauss OJ, Warren RM, Jordaan A, Streicher EM, Hanekom M, Falmer AA Albert H, Trollip A, Hoosain E, van Helden PD, et al: Spread of a low-fitness drug-resistant Mycobacterium tuberculosis strain in a setting of high human immunodeficiency virus prevalence. J Clin Microbiol 2008, 46(4):1514-1516.

48. Sreevatsan S, Pan X, Stockbauer KE, Williams DL, Kreiswirth BN, Musser JM: Characterization of rpsL and rrs mutations in streptomycin-resistant Mycobacterium tuberculosis isolates from diverse geographic localities. Antimicrob Agents Chemother 1996, 40(4):1024-1026.

49. Via LE, Cho SN, Hwang S, Bang H, Park SK, Kang HS, Jeon D, Min SY, Oh T, Kim Y, et al: Polymorphisms associated with resistance and crossresistance to aminoglycosides and capreomycin in Mycobacterium tuberculosis isolates from South Korean Patients with drug-resistant tuberculosis. J Clin Microbiol 2010, 48(2):402-411.

50. Suzuki Y, Katsukawa C, Tamaru A, Abe C, Makino M, Mizuguchi Y, Taniguchi $\mathrm{H}$ : Detection of kanamycin-resistant Mycobacterium tuberculosis by identifying mutations in the 16S rRNA gene. J Clin Microbiol 1998, 36(5):1220-1225.

51. Aubry A, Veziris N, Cambau E, Truffot-Pernot C, Jarlier V, Fisher LM: Novel gyrase mutations in quinolone-resistant and -hypersusceptible clinical isolates of Mycobacterium tuberculosis: functional analysis of mutant enzymes. Antimicrob Agents Chemother 2006, 50(1):104-112.

52. Sreevatsan S, Stockbauer KE, Pan X, Kreiswirth BN, Moghazeh SL, Jacobs WR Jr, Telenti A, Musser JM: Ethambutol resistance in Mycobacterium tuberculosis: critical role of embB mutations. Antimicrob Agents Chemother 1997, 41(8):1677-1681.

53. Johnson R, Jordaan AM, Pretorius $L$, Engelke E, van der Spuy G, Kewley C, Bosman $M$, van Helden PD, Warren R, Victor TC: Ethambutol resistance testing by mutation detection. Int J Tuberc Lung Dis 2006, 10(1):68-73.

54. Sreevatsan S, Pan X, Zhang Y, Kreiswirth BN, Musser JM: Mutations associated with pyrazinamide resistance in pncA of Mycobacterium tuberculosis complex organisms. Antimicrob Agents Chemother 1997, 41(3):636-640.

55. Cheng SJ, Thibert L, Sanchez T, Heifets L, Zhang Y: pncA mutations as a major mechanism of pyrazinamide resistance in Mycobacterium tuberculosis: spread of a monoresistant strain in Quebec, Canada. Antimicrob Agents Chemother 2000, 44(3):528-532.

56. Mphahlele M, Syre H, Valvatne $H$, Stavrum R, Mannsaker T, Muthivhi T, Weyer K, Fourie PB, Grewal HM: Pyrazinamide resistance among South African multidrug-resistant Mycobacterium tuberculosis isolates. J Clin Microbiol 2008, 46(10):3459-3464.

57. Felsenstein J: PHYLIP (Phylogeny Inference Package) version 5.3c. Department of Genetics, University of Washington, Seattle; 1993.

58. Johnson R, Warren R, Strauss OJ, Jordaan AM, Falmer AA, Beyers N, Schaaf HS, Murray M, Cloete K, van Helden PD, et al: An outbreak of drugresistant tuberculosis caused by a Beijing strain in the western Cape, South Africa. Int J Tuberc Lung Dis 2006, 10(12):1412-1414.

59. Warren RM, Victor TC, Streicher EM, Richardson M, van der Spuy GD, Johnson R, Chihota VN, Locht C, Supply P, van Helden PD: Clonal expansion of a globally disseminated lineage of Mycobacterium tuberculosis with low IS6110 copy numbers. J Clin Microbiol 2004, 42(12):5774-5782.

60. Victor TC, Streicher EM, Kewley C, Jordaan AM, van der Spuy GD, Bosman M, Louw H, Murray M, Young D, van Helden PD, et al: Spread of an emerging Mycobacterium tuberculosis drug-resistant strain in the western Cape of South Africa. Int J Tuberc Lung Dis 2007, 11(2):195-201.

61. Pillay M, Sturm AW: Evolution of the extensively drug-resistant F15/ LAM4/KZN strain of Mycobacterium tuberculosis in KwaZulu-Natal, South Africa. Clin Infect Dis 2007, 45(11):1409-1414.

62. loerger TR, Koo S, No EG, Chen X, Larsen MH, Jacobs WR Jr, Pillay M, Sturm AW, Sacchettini JC: Genome analysis of multi- and extensivelydrug-resistant tuberculosis from KwaZulu-Natal, South Africa. PLoS One 2009, 4(11):e7778.
63. Parwati I, van Crevel R, van Soolingen D: Possible underlying mechanisms for successful emergence of the Mycobacterium tuberculosis Beijing genotype strains. Lancet Infect Dis 2010, 10(2):103-111.

64. Ebrahimi-Rad M, Bifani P, Martin C, Kremer K, Samper S, Rauzier J, Kreiswirth B, Blazquez J, Jouan M, van Soolingen D, et al: Mutations in putative mutator genes of Mycobacterium tuberculosis strains of the WBeijing family. Emerg Infect Dis 2003, 9(7):838-845.

65. Durbach SI, Springer B, Machowski EE, North RJ, Papavinasasundaram KG, Colston MJ, Bottger EC, Mizrahi V: DNA alkylation damage as a sensor of nitrosative stress in Mycobacterium tuberculosis. Infect Immun 2003, 71(2):997-1000.

66. Dos Vultos T, Blazquez J, Rauzier J, Matic I, Gicquel B: Identification of Nudix hydrolase family members with an antimutator role in Mycobacterium tuberculosis and Mycobacterium smegmatis. J Bacteriol 2006, 188(8):3159-3161.

67. Werngren J, Hoffner SE: Drug-susceptible Mycobacterium tuberculosis Beijing genotype does not develop mutation-conferred resistance to rifampin at an elevated rate. J Clin Microbiol 2003, 41(4):1520-1524.

68. Calver AD, Falmer AA, Murray M, Strauss OJ, Streicher EM, Hanekom M, Liversage T, Masibi M, van Helden PD, Warren RM, et al: Emergence of increased resistance and extensively drug-resistant tuberculosis despite treatment adherence, South Africa. Emerg Infect Dis 2010, 16(2):264-271.

doi:10.1186/1471-2164-11-670

Cite this article as: loerger et al:: The non-clonality of drug resistance in Beijing-genotype isolates of Mycobacterium tuberculosis from the Western Cape of South Africa. BMC Genomics 2010 11:670.

\section{Submit your next manuscript to BioMed Central and take full advantage of:}

- Convenient online submission

- Thorough peer review

- No space constraints or color figure charges

- Immediate publication on acceptance

- Inclusion in PubMed, CAS, Scopus and Google Scholar

- Research which is freely available for redistribution

Submit your manuscript at www.biomedcentral.com/submit
C) Biomed Central 